\title{
Pancreatic Incidentaloma: take it or leave it?
}

\author{
Akkaraphorn Deeprasertvit* \\ Police General Hospital, Thailand
}

Received: May 25, 2018; Published: June 06, 2018

*Corresponding author: Akkaraphorn Deeprasertvit, HPB Division, Department of Surgery, Police General Hospital, 492/1, Rama I Road, Pathumwan, Bangkok, Thailand

\begin{abstract}
"Incidentaloma" has been generally used to describe an asymptomatic lesion incidentally detected by imaging studies or biochemical diagnostic tests. The first report about "Pancreatic Incidentaloma" (PI) was published in Russian literature in 2001. Incidental pancreatic cysts series have been gradually reported since then. Nowadays we have better developed radiologic studies such as ultrasonography, CT, and MRI, which have been widely used. This explains how we could detect more PIs. The main issues we are concerned with are the lesion characteristics if we have options to safely observe or to remove it, which depends on risk of malignancy. Choosing proper investigations for PI is a challenging issue to take the risk of either surgery or untreated pancreatic cancer. However, despite decreased operative risk due to better technologies and operative techniques, postoperative morbidity is still as high as $40 \%$. The basic principle for preoperative assessment consists of avoidance of unnecessary surgery, especially when the lesion is less likely to be malignant, and respectability determination, if the lesion contains malignant features. The patient's information, history taking, physical examination, investigations (pancreatic protocol CT/MRI/MRCP) play an important role in making a diagnosis. Besides these, there are many other useful tools, such as serum markers, endoscopic ultrasound, and staging laparoscopy. Pancreatic mass workup remains a challenging clinical task, but can be approached by a thoughtful structural approach.
\end{abstract}

Abbreviations: PI: Pancreatic Incidentaloma; FDA: Food and Drug Administration; ASCO: American Society of Clinical Oncology; AIP: Auto Immune Pancreatitis; OS: Overall Survival; PFS: Progression-Free Survival; US: Ultrasonography; MDCT: Multiple Detector Computed Tomography; SMA: Superior Mesenteric Artery; EUS: Endoscopy Ultrasonography

\section{Introduction}

Incidentaloma is defined as asymptomatic lesions found during imaging or biochemical diagnosis. Unlike increasingly diagnosed pancreatic incidentaloma (PI), there are clear protocols for diagnosis and treatments of adrenal gland incidentaloma [1,2]. The first PI case was a Russian literature reported by Kostiuk in 2001 [3]. Later, two incidental pancreatic cyst series were reported [4,5]. In 2006, Winter et al. reported large series of pancreaticoduodenectomies performed for pancreatic head and periampullary incidentalomas, however, these reports did not include incidentalomas at the more frequently found body and tail of pancreas [6,7]. More PI cases were then reported after that [7-9]. Recently, a guidance review about diagnosis and treatments for pancreatic neuroendocrine incidentalomas by Herrera et al. has been reported [10]. Since development of imaging technology and usage of imaging technology for screening test have increased, more PI cases have also been reported. Approximately $6-23 \%$ of all cases of pancreatic surgery were asymptomatic pancreatic lesions $[6,11]$. They were also incidentally found in patients with genitourinary and renal symptoms (61\%), those who undergone asymptomatic abnormal liver function tests (13\%), screening or surveillance (7\%), and those with chest pain (6\%) [7] Solid and cystic lesions were found at $48 \%$ and $52 \%$, respectively, at distal pancreatic (56\%) and head pancreatic (44\%) sites [8]. Several PI cases reported that malignant lesions were found at approximately $30 \%$, and premalignant lesions were found at 50-60\% [6-8]. Since the majority of lesions are premalignant, surgical resection is required. Compared with a symptomatic disease, an early detection and surgery of incidental pancreatic lesions result in higher cure rate and long-term survival rate [6].

\section{Biomarkers}

\section{Carbohydrate antigen 19-9 (CA 19-9)}

Serum carbohydrate antigen 19-9 (CA 19-9) is the only approved antigen testing in blood by the Food and Drug Administration (FDA) for diagnosis of pancreatic cancer. However, detection of CA 19-9 is not very effective for pancreatic cancer screening with only $70 \%$ sensitivity, $87 \%$ specificity, $59 \%$ positive predictive value, $92 \%$ negative predictive value, and $84 \%$ accuracy [12]. As a result, this technique is not recommended to be used for pancreatic cancer screening since $5-10 \%$ of population lack glycosyl transferase 
Lewis blood group antigen, which is necessary for expression of CA 19-9 [13]. Moreover, false-positive could be detected in benign disease or obstructive jaundice state, hence; CA 19-9 detection should be interpreted after biliary decompression. The American Society of Clinical Oncology (ASCO) does not recommend to detect CA 19-9 alone as a marker for determining resectability of pancreatic cancer, instead it is recommended to use to select patients for staging laparoscopy and measuring recurrent after surgical resection [14]. Lack of CA 19-9 response to chemotherapy was a strong negative predictor of survival.

\section{Chromogranin A}

Chromogranin A that is collected and secreted from neuroendocrine cell vesicles can be used as a tumour marker for neuroendocrine tumour diagnosis [10]. This antigen can be detected in both nonfunctioning and functioning tumours together with some specific hormones e.g. insulin, C-peptide, pro-insulin, gastrin, glucagon, vasoactive intestinal peptide, parathyroid hormone-related protein, adrenocorticotropic hormone, and somatostatin, depending on clinical manifestations $[15,16]$. Nonetheless, there can be false-positive results due to common clinical conditions which are decreased renal function, under proton pump inhibitors treatment, and essential hypertension [17]. Sensitivity of chromogranin A detection is between $77.8 \%-84 \%$ and specificity from $71.3 \%-85.3 \%$ depending on techniques used for the detection [18].

\section{Immunoglobulin G4 (IgG4)}

Serum immunoglobulin G4 (IgG4) is an autoimmune pancreatitis (AIP) marker. Previous studies reported that serum IgG4 was precisely used for distinguishing AIP from pancreatic cancer and other autoimmune diseases (sensitivity from $50 \%$ to $92 \%$ and specificity over 90\%) [19]. Frulloni et al. detected IgG in AIP compared with pancreatic cancer, and found $95 \%$ of antibody in AIP and $10 \%$ of antibody in pancreatic cancer, making it an imperfect test to distinguish between the two conditions [20]. IgG4 has found to be useful as a marker for steroid treatment efficacy, in which a decrease in IgG4 serum concentrations was found in 4 of 7 evaluated studies [21].

\section{Novel Serum Marker}

The purpose of discovery of novel biomarkers is to be able to assist in discrimination of benign lesions from malignant lesions with sensitivity and specificity more than those of CA 19-9 detection. There have been several studied novel biomarkers such as MUC1, MUC4, CEACAM1, and MIC-1 that need to be studied further [22]. Using biomarker panels instead of single biomarker for research helps to increase sensitivity and specificity for PI diagnosis.

\section{Circulating Tumor Cells and DNA/RNA Markers}

In peripheral blood of cancer patient, there have been reported of circulating tumour cells (CTCs). CTCs can be detected as low amount as one cell in ten million blood cells, which is a promise biomarker for evaluation of pancreatic cancer prognosis and response to treatment [23]. Nevertheless, there has been no test that has sufficient sensitivity and can reliably measure a significant amount of CTCs at an early stage of disease. Jones et al. [22] conducted a study which had the largest set of gene expressions in pancreatic cancer. They found that 541 genes in $90 \%$ of pancreatic cancer patients had 10-fold increase in expressions compared with normal cells. Among the 541 genes, they are CDKN2A, SMAD4, P53 (tumour suppressor genes), and KRAS (oncogene). Liu et al. [24] studied expressions of microRNAs (e.g. R-20a, R-21, R-24, R-25, R-99a, R-185 and R-191) for screening of pancreatic cancer patients from patients with benign tumour. They found that expressions of microRNAs were found in $90 \%$ of pancreatic cancer patients while only CA 19-9 and CEA were found only $60 \%$ and $30 \%$, respectively. Moreover, miR-21 was found to have highest association with survival of pancreatic cancer patients [25]. A study by Giovannetti et al. reported that higher level of miR-21 in patients with metastatic pancreatic cancer associated with lower progression-free survival (PFS) and overall survival (OS). Similar results were found in patients underwent adjuvant chemotherapy [26].

\section{Radiology \\ Ultrasonography (US)}

Transabdominal ultrasonography is an easy and inexpensive technique that can be used for preliminary detection of non-invasive cancer. Bowel gas is the major interference and makes it difficult for the evaluation, with the sensitivity range from $50 \%$ to $90 \%$ [27]. For pancreatic cancer diagnosis, the technique is uncertain because it is operator-dependent. Characteristics of pancreatic cancer seen from the US are hypoechoic mass, dilation of pancreatic duct, and dilation of bile duct.

\section{Computer Tomography (CT)}

Multiple detectors computed tomography (MDCT) had 90\% sensitivity, 96\% specificity, and 80-90\% accuracy, which are high for diagnosis of pancreatic cancer. This technique is also useful in identification of tumour position, evaluation of resectability, peritumoural organ invasion and vascular invasion. Blood vessels in those areas are celiac artery, superior mesenteric artery (SMA), superior mesenteric vein (SMV), splenic vein, and portal vein. Protocol for pancreatic imaging is $1 \mathrm{~mm}$ slice of thickness with 3 phases which are arterial phase at the 17-25s, late arterial at the 35-50s, and portovenous phase at 55-70s. However, discrimination between inflammatory and neoplastic characteristics in lesions smaller than 2 $\mathrm{cm}$ is sometimes equivocal [28].

\section{Magnetic Resonance Imaging (MRI)}

MRI and magnetic resonance cholangiopancreatography (MRCP) provides information similar to a contrast enhanced CT with $93 \%$ sensitivity and $75 \%$ specificity for pancreatic cancer detection. MRCP can detect double duct sign (dilation of CBD and main pancreatic duct) up to $75 \%$ [29]. It is a non-invasive technique that gives detailed physical information of biliary tract, suitable for diagnosing the cause of painless obstructive jaundice in patients. MRCP can detect $95-100 \%$ of constricted biliary tract cases.

\section{Positron Emission Tomography (PET) Scan}

PET scan has $86.4 \%$ sensitivity and $78.9 \%$ specificity. This technique can detect liver, lungs, or bone micrometastasis [30]. It 
is preferred to CT scan when searching for lesion smaller than 2 $\mathrm{cm}$, however, CT scan is preferred to PET scan for searching lesion larger than $4 \mathrm{~cm}$ because of a lower metabolic rate in a larger lesion. PET scan in combination with other imaging techniques such as CT or MRI increases diagnostic efficiency of pancreatic cancer. Nagamachi et al. compared PET/MRI with PET/CT for pancreatic cancer diagnosis. The results showed that PET/MRI had higher accuracy (96.6\% compared with $86.6 \%$ ), especially in solid tumour $(97.7 \%$ compared with $85.2 \%$ ). Though, there were some false-positive results from patients with DM or other conditions resulting in high glucose uptake [31].

\section{Endoscopy}

Endoscopy ultrasonography (EUS) is a safe and efficient technique with sensitivity and specificity higher than $90 \%$ [32]. It is a non-invasive technique, so it does not cause cancer to spread into peritoneal cavity. It also helps to better evaluate vascular and local invasion than other modality with some limitation in patients with chronic pancreatitis, in which the sensitivity is lower than $75 \%$. EUS can be used in combination with other techniques to increase its efficiency. For example, contrast injection is used while performing EUS called contrast enhancement EUS (CE-EUS), and elastrography is used to increase EUS capacity since tumours with malignant tendency usually are dense and less elastic than benign tumours or normal pancreatic tissue. Fine needle aspiration biopsy (EUSFNAB) can also be used to acquire cells for examination. EUS can be used as a guide in biopsy for molecular examination to identify molecular or genetic markers associated with cancer development. Sensitivity and specificity of EUS-FNAB are 50-70\% and 70-100\%, respectively. When used in combination with other techniques, the sensitivity and specificity of EUS increase especially CE-EUS.

The indications for EUS are making diagnosis and decision in order to proceed surgery, unresectable cancer requiring chemotherapy or radiation, and exclusion of other cancers that may require a different management. On the other hand, contraindications of EUS are unaffected management by FNA result, inability to visualize a lesion, and the position of involved vessel between gut and the target [33]. Bournet et al. performed a prospective multicentre study on EUS-guided FNAB examination with KRAS mutation assay in order to separate pancreatic cancer from pancreatitis with mass in 178 patients with solid pancreatic mass from imaging study. The patients received chemotherapy or undergone pancreatic surgery or under any interdictions for EUS were excluded from the study. Diagnosis was confirmed by pathology result of the tumour gained after surgery, clinical follow up, or repeating EUS-FNAB. The results showed that detection of KRAS mutation increased accuracy in the diagnosis. Regarding subgroup analysis, KRAS mutation detection was beneficial in case the indiscrimination of cancer in suspicious normal pancreatic tissues. Twenty nine patients who failed to be diagnosed by FNAB, KRAS mutation were confirmed in seven of those patients and they were confirmed to be chronic pancreatitis in the later diagnosis.

Twenty-seven patients with wild-type KRAS all had chronic pancreatitis, and the follow-up showed no sign of cancer. As a result, patients with benign tumor with wild-type KRAS might avoid the unnecessary surgery $[34,35]$. CE-EUS increases diagnostic efficiency of pancreatic cancer. Several contrast agents have been used with different binding and detecting capacity by EUS. Important properties would be a gaseous state at room temperature and safe to the patients. Sensitivity and specificity of this technique are $94 \%$ and $89 \%$, respectively [36]. Small vessels in the tumour and the surrounding areas can be seen clearly, so the lower signal hypovascular lesion and hypervascular lesion can be detected in the pancreatic cancer and pancreatic neuroendocrine tumour (pNET), respectively, compared with normal tissues. A prospective multicenter trial conducted by Arcidiacono P in 258 patients with focal pancreatic mass from 13 European medical centers showed that EUS elastrography assisted in detecting pancreatic cancer with good accuracy (85.4\%) and high sensitivity (93.4\%), however, the specificity was only $66 \%$ due to the difficulty in controlling tissue compression. If the probe was pressed too hard, the results could be misinterpreted [37].

EUS-guided fine needle aspiration for analysis of cyst fluid is used for distinguishing between mucinous and nonmucinous cysts. Cyst fluid can be analyzed for tumour markers (CEA, CA 72-4, CA 125, CA 19-9, and CA 15-3), cytology, mucins, DNA analysis and amylase. Pancreatic cyst CEA level is considered the most accurate tumour marker for diagnosing mucinous cysts. A cut-off of $192 \mathrm{ng} /$ $\mathrm{mL}$ can be expected to capture $\sim 75 \%$ of mucinous cysts [38]. Cytology is especially helpful in diagnosing malignancy typically in the presence of a solid component in the cyst. Cytology can help in diagnosis of; 1) MUC-containing cells found in intraductal papillary mucinous neoplasms (IPMN) and mucinous cystic neoplasms (MCN), 2) malignant cells, 3) glycogen-rich cuboidal cells found in serous cystic tumour, 4) branching papillae with myxoid stroma found in solid pseudopapillary neoplasm (SPN), and 5) abundant anucleate squamous cells and debris in lymphoepithelial cysts. Khalid et al. performed the prospective, multicentre study of pancreatic cyst DNA analysis. They found that the presence of a KRAS mutation in cyst fluid was highly specific for a mucinous cyst (96\%) but the sensitivity (45\%) was low [39]. Amylase is an indicator of pancreatic duct communication. High levels are most commonly seen in pseudocysts, MCN, and IPMN.

\section{Management}

PI lesions could be detected by imaging modalities, biomarkers tests, or endoscopic evaluation. Distinguishing lesion characteristics between benign and malignant potential ones is important for treatment decision making. The principle of PI lesions treatment is to balance between the risk of surgical resection and untreated pancreatic cancer. As nowadays there is still no qualified isolated diagnostic test. A multimodal approach, which is a combination of clinical presentation e.g. history of jaundice, anorexia, previous pancreatitis, new onset of diabetes mellitus, decreasing BMI, biomarkers, imaging, and endoscopic studies is essential for making diagnosis.

\section{Management of Solid Lesions}

In most PI series, solid lesions could be found as much as cystic ones. Benign solid PIs are focal pancreatitis, lipomatosis, and benign neuroendocrine tumours. Malignant lesions are pancreatic 
ductal adenocarcinoma, malignant neuroendocrine tumours, rare malignancies, and metastasis. Bruzoni et al. collected PI series in 365 patients, $61 \%$ were solid lesions, $54 \%$ were malignant, and almost $20 \%$ were premalignant e.g. neuroendocrine tumors, borderline IPMN, pseudopapillary tumours and mucinous cystadenomas [40]. As well as Winter et al. and Sachs et al. series, which solid PIs have high malignant potential [6,7]. Solid lesions are more likely to be malignant or premalignant. Surgery is therefore mandated if there's no contraindication, once lesions are detected. Lahat et al. studied about series of pancreatectomies in 475 patients. Sixty-four patients $(13.5 \%)$ of pancreatectomies underwent a surgical resection for a PI. 94\% have malignant potential, which 34\% were malignant and $60 \%$ were premalignant respectively [8]. The extent of surgery in solid PIs depends on tumours size, location, number of lesions and probable diagnosis. Oncologic resection was considered in malignant cases or those, which malignancy cannot be ruled out. Regarding tumours location, either pancreaticoduodenectomy or distal pancreatectomy can be performed. One study reported resected malignant PIs have favorable pathologic features comparing to resected malignant non incidental pancreatic tumours. Early PI lesions had better survival rate than symptomatic ones [6]. The management of incidental detection of pancreatic neuroendocrine tumours (PNETs) varies according to size and nature of the tumours. Poorly differentiated PNETs usually have metastasis and rarely resectable. Well or intermediately-differentiated tumours, size at least $2 \mathrm{~cm}$, with imaging evidence of malignancy or Ki-67 more than $2 \%$, should be resected. Non-MEN related pNETs, nonfunctioning ones, and asymptomatic ones, size smaller than $2 \mathrm{~cm}$, with Ki-67 index $\leq 2 \%$, are considered as low risk of metastasis. Those can be observed as long as they are asymptomatic, not in radiologic criteria of malignancy or progressive disease, especially in elderly patients [10]. Nevertheless, physicians need to consider the patient's age, lesion location, and operative risks, before choosing the treatment. Close follow-up should be done in the patients not receiving surgery. Enucleation is suitable for pNETs, size smaller than $2 \mathrm{~cm}$, located superficially, and without pancreatic duct involvement. Pancreatic resection are recommended in large tumours, locating close to the pancreatic duct, or malignant pNETs $[41,42]$.

\section{Management of cystic lesions}

Cystic PIs can be classified into several types e.g. congenital (true serous cysts and syndromes associated with multiple cysts), inflammatory (pseudocysts, hydatid cysts) and neoplastic (SCNs, MCNs, IPMNs). The most common cystic lesion is pseudocyst and IPMN is the most common neoplastic lesion [43]. Imaging studies, especially MRI play important role in making diagnosis by lesion characteristics. Having solid mass, intramural nodule, main pancreatic duct dilatation, thick septations, and biliary obstruction should be suspicious of malignancy [44]. According to the international consensus guidelines 2012 for management of IPMN and MCN of the pancreas, cystic lesion with no worrisome or high-risk features can be follow-up with ultrasonography, MRI/MRCP or EUS depending on diagnosis and cyst size [45]. EUS should be done in cyst with worrisome features $(>3 \mathrm{~cm}$, thickened enhancing wall, non en- hancing mural nodule, main pancreatic duct 5-9 mm). Pancreatic resection is recommended in PIs with mural nodules, main pancreatic duct involvement or cytology positive for malignancy. In case of high-risk stigmata, size larger than $1 \mathrm{~cm}$, with enhancing solid component, main pancreatic duct at least $10 \mathrm{~mm}$, tumours should be resected.

\section{Conclusion}

The work-up of PIs is a challenging clinical problem in which the risk of surgical resection must be weighed against untreated pancreatic cancer. Nowadays morbidity from pancreatic resection is still as high as $20 \%$, even when mortality is lower than $5 \%$. As mentioned earlier, a more precise diagnosis could be achieved by the multimodal approach, as a combination of clinical presentation especially cancer-related, serum markers, and imaging studies e.g. CT, MRI/MRCP, EUS. Even though definitive diagnosis could not be made, PIs work-up is still particularly challenging for clinicians.

\section{References}

1. Udelsman R, Fishman EK (2000) Radiology of the adrenal. Endocrinol Metab Clin North Am 29(1): 27-42.

2. Thompson GB, Young WF Jr (2003) Adrenal incidentaloma. Curr Opin Oncol 15(1): 84-90.

3. Kostiuk TS (2001) Observation of pancreatic incidentaloma. KlinKhir 9: 62-63.

4. Fernandez-del Castillo C, Targarona J, Thayer SP, Rattner DW, Brugge WR, et al. (2003) Incidental pancreatic cysts: clinicopathologic characteristics and comparison with symptomatic patients. Arch Surg 138(4): 427-423.

5. Handrich SJ, Hough DM, Fletcher JG, Sarr MG. et al. (2005) The natural history of the incidentally discovered small simple pancreatic cyst: longterm follow-up and clinical implications. AJR Am J Roentgenol 184(1): 20-23.

6. Winter JM, Cameron JL, Lillemoe KD, Campbell KA, Chang D, et al. (2006) Periampullary and pancreatic incidentaloma: a single institution's experience with an increasingly common diagnosis. Ann Surg 243(5): 673-683.

7. Sachs T, Pratt WB, Callery MP, Vollmer CM Jr (2009) The Incidental Asymptomatic Pancreatic Lesion: Nuisance or Threat? J Gastrointest Surg 13(3): 405-415.

8. Lahat G, Haim MB, Nachmany I, Sever R, Blachar A, et al. (2009) Pancreatic Incidentalomas: High Rate of Potentially Malignant Tumors. J Am Coll Surg 209(3): 313-319.

9. Karatzas T, Dimitroulis D, Charalampoudis P, EP Misiakos, I Vasileiadis, et al. (2013) Management of cystic and solid pancreatic incidentalomas: A review analysis. J Buon 18(1): 17-24.

10. Herrera MF, Åkerström G, Angelos P, Grant CS, Hoff AO, et al. (2015) AACE/ACE Disease State Clinical Review: Pancreatic Neuroendocrine Incidentalomas. Endocr Pract 21(5): 546-553.

11. Kent TS, Vollmer Jr CM, Callery MP (2010) Intraductal papillary mucinous neoplasm and the pancreatic incidentaloma. World J Gastrointest Surg 2(10): 319-323.

12. Fong ZV, Winter JM (2012) Biomarkers in Pancreatic Cancer: Diagnostic, Prognostic, and Predictive. Cancer J 18(6): 530-538.

13. Maithel SK, Maloney S, Winston C, Gönen M, D’Angelica MI, et al. (2008) Preoperative CA 19-9 and the yield of staging laparoscopy in patients with radio- graphically resectable pancreatic adenocarcinoma. Ann Surg Oncol 15(12): 3512-3520. 
14. van den Bosch RP, van Eijck CH, Mulder PG, Jeekel J (1996) Serum CA 19-9 determination in the management of pancreatic cancer. Hepatogastroenterology 43(9): 710-713.

15. Vinik AI, Woltering EA, Warner RR, Caplin M, O'Dorisio TM, et al. (2010) NANETS consensus guidelines for the diagnosis of neuroendocrine tumor. Pancreas 39(6): 713-734.

16. Falconi M, Bartsch DK, Eriksson B, Klöppel G, Lopes JM, et al. (2012) ENETS Consensus Guidelines for the management of patients with digestive neuroendocrine neoplasms of the digestive system: welldifferentiated pancreatic non-functioning tumors. Neuroendocrinology 95(2): 120-134.

17. Nobels FR, Kwekkeboom DJ, Bouillon R, Lamberts SW (1998) Chromogranin A: Its clinical value as marker of neuroendocrine tumours. Eur J Clin Invest 28(6): 431-440.

18. Zatelli MC, Torta M, Leon A, Ambrosio MR, Gion M, et al. (2007) Chromogranin A as a marker of neuroendocrine neoplasia: An Italian Multicenter Study. Endocr Relat Cancer 14(2): 473-482.

19. Hamano H, Kawa S, Horiuchi A, Unno H, Furuya N, et al. (2001) High serum IgG4 concentrations in patients with sclerosing pancreatitis. N Engl J Med 344(10): 732-738.

20. Frulloni L, Lunardi C, Simone R, Dolcino M, Scattolini C, et al. (2009) Identification of a Novel Antibody Associated with Autoimmune Pancreatitis. N Engl J Med 361(22): 2135-2142.

21. Morselli-Labate AM, Pezzilli R (2009) Usefulness of serum IgG4 in the diagnosis and follow up of autoimmune pancreatitis: A systematic literature review and meta-analysis. J Gastroenterol Hepatol 24(1): 1536.

22. Liang JJ, Kimchi ET, Staveley-O'Carroll KF, Tan D (2009) Diagnostic and prognostic biomarkers in pancreatic carcinoma. Int J Clin Exp Pathol 2(1): $1-10$.

23. Torphy RJ, Tignanelli CJ, Kamande JW, Moffitt RA, Herrera Loeza SG, et al. (2014) Circulating Tumor Cells as a Biomarker of Response to treatment in Patient-Derived Xenograft Mouse Models of Pancreatic Adenocarcinoma, Plos One 9(2): e89474.

24. Jones S, Zhang X, Parsons DW, Lin JC, Leary RJ, et al. (2008) Core Signaling pathways in Human Pancreatic Cancers Revealed by Global Genomic Analyses. Sciences 321(5897): 1801-1806.

25. Liu R, Chen X, Du Y, Yao W, Shen L, et al. (2012) Serum MicroRNA Expression Profile as a Biomarker in the Diagnosis and Prognosis of Pancreatic Cancer. Clin Chem 58(3): 610-618.

26. Giovannetti E, Funel N, Peters GJ, Del Chiaro M, Erozenci LA, et al. (2010) MicroRNA-21 in Pancreatic Cancer: Correlation with Clinical Outcome and Pharmacologic Aspects Underlying Its Role in the Modulation of Gemcitabine Activity. Cancer Res 70(11): 4528-4538.

27. Lee ES, Lee JM (2014) Imaging diagnosis of pancreatic cancer: A stateof-the-art review. World J Gastroenterol 20(24): 7864-7877.

28. Yoon SH, Lee JM, Cho JY, Lee KB, Kim JE, et al. (2011) Small ( $\leq 20 \mathrm{~mm}$ ) pancreatic adenocarcinomas: Analysis of enhancement patterns and secondary signs with multiphasic multidetector CT. Radiology 259(2): $442-452$

This work is licensed under Creative Commons Attribution 4.0 License

Submission Link: https://biomedres.us/submit-manuscript.php
29. Griffin N, Charles-Edwards G, Grant LA (2012) Magnetic resonance cholangiography: the ABC of MRCP, European Society of Radiology. Insights Imaging 3(1): 11-21.

30. Dutta AK, Chacko A (2015) Head mass in chronic pancreatitis: Inflammatory or malignant. World J Gastroenterol Endosc 7(3): 258264.

31. Nagamachi S, Nishii R, Wakamatsu H, Mizutani Y, Kiyohara S, et al. (2013) The usefulness of 18F-FDG PET/MRI fusion image in diagnosing pancreatic tumor: comparison with 18F-FDG PET/CT. Ann Nucl Med 27(6): 554-563.

32. Wiersema MJ (2001) Accuracy of endoscopic ultrasound in diagnosing and staging pancreatic carcinoma. Pancreatology 1(6): 625-632.

33. Yamao K, Sawaki K, Mizuno N, Shimizu Y, Yatabe Y, et al. (2005) Endoscopic Ultrasound-guided fine-needle aspiration biopsy (EUSFNAB); past, present, and future. J Gastroenterol 40(11): 1013-1023.

34. Bournet B, Sonque A, Senesse P, Assenat E, Barthet M, et al. (2009) Endoscopic ultrasound-guided fine-needle aspiration biopsy coupled with KRAS mutation assay to distinguish pancreatic cancer from pseudotumoral chronic pancreatitis. Endoscopy 41(6): 552-557.

35. Bournet B, Gayral M, Torrisani J, Janick Selves, Pierre Cordelier, et al. (2014) Role of endoscopic ultrasound in the molecular diagnosis of pancreatic cancer. World J Gastroenterology 20(31): 10758-10768.

36. Jang SI, Lee DK (2014) Contrast-enhanced endoscopic ultrasonography: advance and current status. Ultrasonography 33(3): 161-169.

37. Arcidiacono PG (2012) Advances in endoscopy: Current development in diagnostic and therapeutic endoscopy. Endoscopy 8(1): 48-67.

38. Rockacy M, Khalid A (2013) Update on pancreatic cyst fluid analysis. Ann Gastroenterol 26(2): 122-127.

39. Khalid A, Zahid M, Finkelstein SD, LeBlanc JK, Kaushik N, et al. (2009) Pancreatic cyst fluid DNA analysis in evaluating pancreatic cysts: a report of the PANDA study. Gastrointest Endosc 69(6): 1095-1102.

40. Bruzoni M, Johnston E, Sasson AR (2008) Pancreatic incidentalomas: clinical and pathologic spectrum. Am J Surg 195(3): 329-332.

41. Franco J, Feng W, Yip L, Genovese E, Moser AJ (2010) Non-functional neuroendocrine carcinoma of the pancreas: incidence, tumor biology and outcomes in 2,158 patients. J Gastrointest Surg 14(3): 541-548.

42. Boninsegna L, Partelli S, D’Innocenzio MM, Capelli P, Scarpa A, et al. (2010) Pancreatic cystic neuroendocrine tumors: a different morphological entity associated with a less aggressive behavior. Neuroendocrinology 92(4): 246-251.

43. Conrad C, Fernandez del Castillo C (2013) Preoperative evaluation and management of the pancreatic head mass. J Surg Oncol 107: 23-32.

44. Sarr MG, Murr M, Smyrk TC, Yeo CJ, Fernandez del Castillo C, et al. (2003) Primary cystic neoplasms of the pancreas. Neoplastic disorders of emerging importance current state of the art and unanswered questions. J Gastrointest Surg 7(3): 417-428.

45. Tanaka M, Fernández-del Castillo C, Adsay V, Suresh Chari, Massimo Falconi, et al. (2012) International consensus guidelines 2012 for management of IPMN and MCN of the pancreas. Pancreatology 12: 183197.

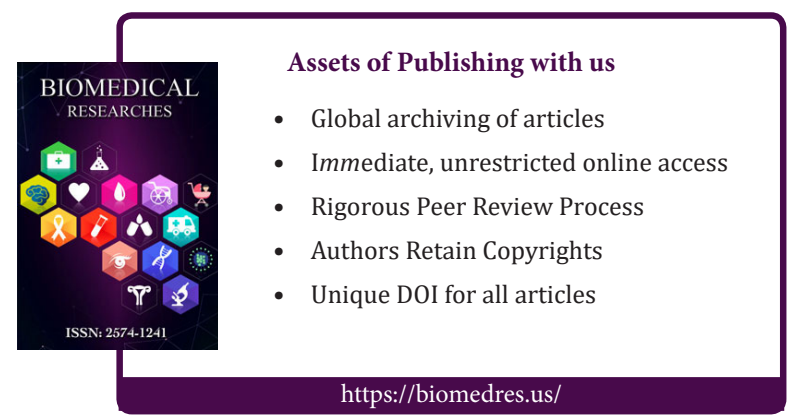

